\title{
Hydatid cyst of the lumbar plexus: case report with review of literature
}

\author{
Ayad Ahmad Mohammed ${ }^{1 *}$ (D) and Wan Aldohuky ${ }^{2}$
}

\begin{abstract}
Background: Hydatid disease is widely distributed worldwide; it is caused by a parasite belonging to the Echinococcus species. It mostly affects the liver and the lungs, involvement of the spinal canal is reported to be around $0.2 \%$, but affection of the peripheral nerves is extremely rare.

Case presentation: A 42-year-old lady was complaining from left loin pain and backache, with pain in the outer aspect of the left thigh for the last 5 years. During clinical examination, the ankle and the knee reflexes were normal and there was no muscle wasting. There was hyperesthesia on the outer aspect of the left leg.

Magnetic resonance imaging of the back demonstrated a cystic lesion between the 4th and the 5th lumbar vertebrae which was pushing the psoas muscle anteriorly and causing atrophy of the muscle. The patient received anthelminthic medications for 1 month before surgery with little improvement of the clinical and the radiological condition.

During surgery the cyst was found to arise from the lumbar nerve sheath and was apparent to be hydatid cyst because it was containing small daughter cysts. Isolation of the cyst was done and complete evacuation with deroofing of the cyst was done. The cavity of the cyst was irrigated with a solution of chlorhexidine as a scolicidal agent. The patient was discharged home after 3 days with no post-operative complications, anthelminthic medications prescribed for 3 months.

Conclusion: The treatment options vary depending on the region affected by the cyst; it may be treated by complete cyst excision, evacuation, and deroofing. Preventing spillage of the contents of the cyst during surgery and the use of scolicidal agents are mandatory to decrease the rate of recurrence. It is very important to exclude other organ involvement particularly pulmonary involvement before surgery.
\end{abstract}

Keywords: Hydatid disease, Echinococcus granulosus, Backache, Lumbar plexus, Scolicidal agents

\section{Introduction}

Hydatid disease is widely distributed worldwide; it is caused by a parasite belonging to the Echinococcus species. The disease is transmitted by the fecal-oral route. It can affect many organs and anatomical sites; the most affected organs are the liver and the lungs. Involvement of the spinal canal is reported to be around $0.2 \%$, but affection of the peripheral nerves is extremely rare [1].

The parasite is transmitted to the para-spinal space by the blood through the direct porto-vertebral venous shunts, the cyst may be variable in size and the

\footnotetext{
* Correspondence: ayad.mohammed@uod.ac

${ }^{1}$ Department of Surgery, College of Medicine, University of Duhok, Azadi

Teaching Hospital, 8 Nakhoshkhana Road, Duhok City, Kurdistan Region 1014

AM, Iraq

Full list of author information is available at the end of the article
}

symptoms and the signs depend on the anatomical site and the size of the cyst [1].

Magnetic resonance imaging is the investigation of choice in detecting hydatid cysts affecting the nerves or the spinal canal. Most cases are treated using the surgical approach; surgery can be done through the open or the minimally invasive techniques. Anthelminthic medications are indicated before surgery or after surgery to prevent recurrence of the cyst $[2,3]$.

The cyst may be completely asymptomatic for long time and may be discovered accidentally, or may cause a very wide range of clinical presentations depending on the site and the size of the cyst. Emergency presentations may occur; even sudden deaths have been reported due to cyst rupture and anaphylactic reaction to the cyst contents [4]. 


\section{Case presentation}

A 42-year-old lady was complaining from left side loin pain and backache, with pain in the outer aspect of the left thigh for the last 5 years. The pain at start was mild and not interfering with the sleep and the daily activities and was relieved with oral acetaminophen and nonsteroidal anti-inflammatory medications. For the last 6 months the pain intensity increased and was interfering with sleeping and walking which was decreased by the intravenous or intramuscular analgesics; there was limping during walking. The patient's past medical and surgical histories were unremarkable. During clinical examination, the ankle and the knee reflexes were normal and there was no muscle wasting. There was hyperesthesia on the outer aspect of the left leg. Other parts of the clinical examination were normal. The initial clinical diagnosis was consistent with lumbar disc herniation.

The patient was sent for magnetic resonance imaging of the back which demonstrated a cystic lesion measuring $8 \times 6 \mathrm{~cm}$ between the 4th and the 5th lumbar vertebrae which was pushing the psoas muscle anteriorly and causing atrophy of the muscle (Fig. 1).

The differential diagnoses were either hematoma, hydatid cyst, or cystic tumor of the soft tissues. Chest Xray and abdominal ultrasound were normal which were done to exclude other organ involvement.

The complete blood count was and the serological test was negative for hydatid disease.

The patient received anthelminthic medications for 1 month before surgery with little improvement of the clinical and the radiological condition.

During surgery the cyst was found to arise from the lumbar nerve sheath and was apparent to be hydatid cyst because it was containing small daughter cysts. Isolation of the cyst was done with complete evacuation and deroofing of the cyst. The cavity of the cyst was irrigated with a solution of chlorhexidine as a scolicidal agent (Figs. 2 and 3).

The patient was discharged home after 3 days with no post-operative complications, anthelminthic medications were prescribed for 3 months, and the patient was followed for 6 months with monthly abdominal ultrasound with no signs of recurrence.

\section{Discussion}

The most common site of hydatid cyst involvement is the region of the abdomen whether the intra-abdominal organs, the retroperitoneum, or the abdominal wall; other locations are affected to lesser frequency like the heart, or the intracranial space, or the soft tissues of the limbs [5].

In a review of literature, there are reported cases that involved the spinal canal, the paravertebral muscles, and the psoas muscle; nerve sheath involvement has been reported but very rarely. Such cases present with various types of clinical presentations; one case reported with features with femoral nerve weakness, and other cases presented with recurrent cyst involving the region of the psoas muscle causing pain in the loin. The cyst may also enlarge alongside the psoas sheath and present as a mass in the iliac fossa or the groin; when the mass reach a large size, it may present as bulging in the loin, or a palpable subcutaneous mass if there is extension to the superficial tissue planes. All the other reported cases were presented with gradually increasing pain; the site of the pain was either in the lower back, loin pain, or abdominal pain with variable degrees of sensory and/or motor deficits affecting the region of the affected nerve roots. Very rarely the enlarged cyst may cause pressure effects on the blood vessels causing clinical features of arterial insufficiency or venous stasis in the affected limb. The serology was negative in most of the reported

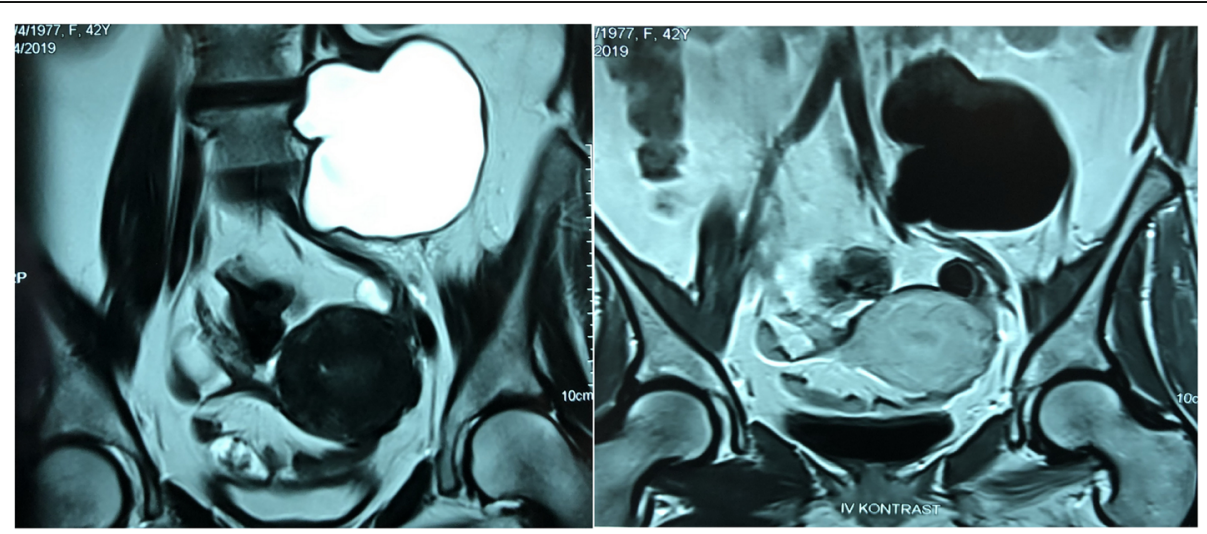

Fig. 1 MRI of the of the lumbar region showing evidence of cystic lesion in the left paravertebral region pressing the psoas muscle and causing atrophy of it 


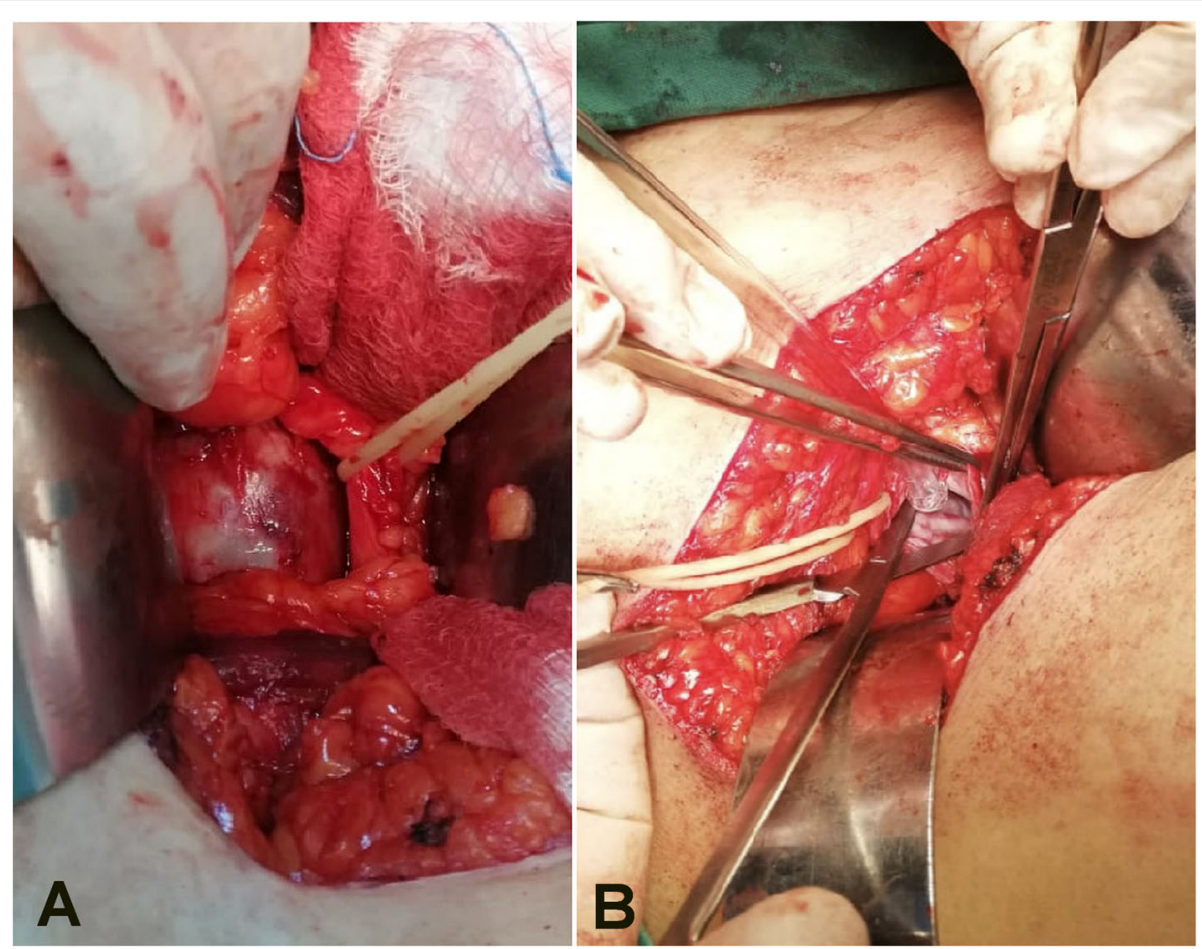

Fig. 2 An intraoperative picture showing a the hydatid cyst and lumbar nerve root, traction is exerted over the nerve. The surgical field is isolated using a surgical pack soaked with chlorhexidine; $\mathbf{b}$ the hydatid cyst cavity is opened containing small daughter cysts

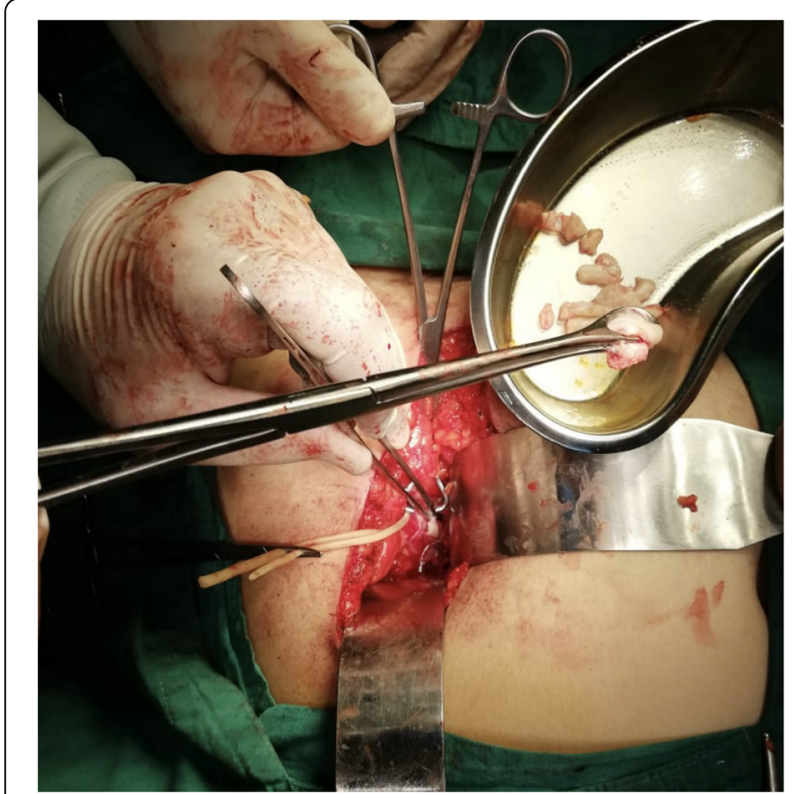

Fig. 3 An intraoperative picture showing the hydatid cyst and the daughter cyst extracted from its cavity cases, and the diagnosis was done either with $\mathrm{CT}$ scan or MRI or done during surgery [1, 3, 6-12].

It is very important, before any kind of surgical intervention, to exclude other organ involvement particularly pulmonary involvement; when pulmonary involvement is detected, it has the priority for management provided that cysts in other sites are not causing any kind of emergency situations. The surgery can be done through the retroperitoneal approach or the transabdominal approach; the retroperitoneal approach is preferred to avoid affection of the intraperitoneal cavity. The treatment options may include complete cyst excision, evacuation, and deroofing. When the cyst is affecting the vertebra or causing vertebral destruction, the treatment includes removal of the affected vertebra with posterior stabilization. Preventing spillage of the contents of the cyst during surgery and the use of scolicidal agents are mandatory during surgery to decrease the rate of recurrence; many types of scolicidal agents have been tried such as hypertonic saline, povidone iodine, chlorhexidine, and diluted solution of formaldehyde $[4,13,14]$.

Although radical excision of the cyst is the best treatment option, sometimes it may not be very easy especially in difficult anatomical sites or in case of recurrent disease in which the second surgery may be very difficult; in these cases the other option of treatment is the nonsurgical approach such as the PAIR technique; this is the abbreviation 
of the following: $\mathrm{P}$, percutaneous injection under radiologic guidance; A, aspiration of some of the cyst contents; I, injection of one of the above mentioned scolicidal solutions; and $\mathrm{R}$, re-aspiration of the contents. The scolicidal solution should remain in the cyst cavity for at least 15 min to ensure death of all the scolices; this technique is applicable for uni-vesicular cysts, the success rate of this technique is variable, and it should be done only for cases where the surgery is difficult or contraindicated [14].

Recurrence of the disease is a problem and it varies among various anatomical sites; recurrence in the paravertebral region is not well reported due to rarity of the infection at this site. Most authors recommend the use of anthelminthic medications before surgery and continued for few months after surgery; this has been shown to significantly reduce the recurrence rate when compared with the results of surgery alone [15].

\section{Acknowledgements}

Not applicable.

\section{Authors' contributions}

Both authors contributed to the concept of reporting the case, writing, and design of work. Both authors read and approved the final manuscript.

\section{Funding}

The authors are the source of funding.

Availability of data and materials

Not applicable.

Ethics approval and consent to participate

Ethical approval was granted from the department of Surgery at Azadi

Teaching Hospital for reporting this case.

\section{Consent for publication}

An informed written consent was taken from the patient for reporting this case and the accompanying images.

\section{Competing interests}

The authors declare that they have no competing interests.

\section{Author details}

${ }^{1}$ Department of Surgery, College of Medicine, University of Duhok, Azadi Teaching Hospital, 8 Nakhoshkhana Road, Duhok City, Kurdistan Region 1014 AM, Iraq. ${ }^{2}$ Department of Surgery, Duhok Directorate General of Health,

Duhok, Kurdistan Region, Iraq.

Received: 14 August 2019 Accepted: 29 November 2019

Published online: 18 December 2019

\section{References}

1. Sharma N, et al. Primary spinal extradural hydatid cyst. Neurol India. 2003; 51(1):89.

2. Pandey M, Chaudhari MP. Primary hydatid cyst of sacral spinal canal: case report. Neurosurgery. 1997:40(2):407-9.

3. Mohammed AA, Arif SH. Hydatid cyst of the parietal peritoneum. J Pediatr Surg Case Rep. 2019;43:80-2.

4. Arif SH, Mohammed AA. Primary hydatid cyst of the urinary bladder. Bmj Case Rep. 2018;2018:bcr-2018-226341.

5. Prousalidis J, et al. Uncommon sites of hydatid disease. World J Surg. 1998; 22(1):17-22.

6. Bilanović $D$, et al. Lesion of the femoral nerve caused by a hydatid cyst of the right psoas muscle. Srp Arh Celok Lek. 2010;138(7-8):502-5.

7. Kafih, M., et al. Hydatid cyst of the psoas muscle. in Annales de gastroenterologie et d'hepatologie. 1993.
8. Rabii R, et al. Hydatid cyst of the psoas muscle. Apropos of a case. In: Annales d'urologie; 1997.

9. Benchekroun A, et al. Hydatid cyst of the psoas. Report of a case. In: Annales d'urologie; 2001

10. El Malki HO, et al. Hydatid cyst of the psoas muscle. Cahiers d'études et de recherches francophones/Santé. 2007;17(3):177-9.

11. Yörükoglu Y, et al. Primary muscular hydatid cyst causing arterial insufficiency: case report and literature review. Angiology. 1993;44(5):399-401.

12. Sekar $\mathrm{N}$, et al. Primary retroperitoneal hydatid cyst (a report of 3 cases and review of the literature). J Postgrad Med. 1982;28(2):112.

13. Herrera A, Martínez AA, Rodríguez J. Spinal hydatidosis. Spine. 2005;30(21): 2439-44.

14. Mohammed AA, Arif SH. Hydatid cyst of the calf presenting as painless mass; a case report. Int J Surg Case Rep. 2019;60:273-5.

15. Salduz A, et al. Long-term result of treatment for paraspinal and extradural hydatid cyst: a case report. Acta Orthop Traumatol Turc. 2009;43(3):267-71.

\section{Publisher's Note}

Springer Nature remains neutral with regard to jurisdictional claims in published maps and institutional affiliations.

\section{Submit your manuscript to a SpringerOpen ${ }^{\circ}$ journal and benefit from:}

- Convenient online submission

- Rigorous peer review

- Open access: articles freely available online

- High visibility within the field

- Retaining the copyright to your article

Submit your next manuscript at $>$ springeropen.com 